BULLETIN OF THE

AMERICAN MATHEMATICAL SOCIETY

Volume 79, Number 4, July 1973

\title{
BOUNDARY BEHAVIOR OF THE CARATHÉODORY, KOBAYASHI, AND BERGMAN METRICS ON STRONGLY PSEUDOCONVEX DOMAINS IN $C^{n}$ WITH SMOOTH BOUNDARY
}

\author{
BY IAN GRAHAM
}

Communicated by Elias Stein, November 27, 1972

Let $G$ be a bounded domain in $C^{n}$. Let $\Delta$ be the unit disk in $C$. Let $\Delta(G)$ be the set of holomorphic mappings from $G$ to $\Delta$, and $G(\Delta)$ the set of holomorphic mappings from $\Delta$ to $G$. The Carathéodory metric on $G$ (i.e. the infinitesimal form, as in [7] is defined by

$$
F_{C}(z, \xi)=\sup _{f \in \Delta(G)}\left|f_{*}(\xi)\right|=\sup _{f \in \Delta(G)}\left|\sum_{i=1}^{n} \frac{\partial f}{\partial z_{i}}(z) \xi_{i}\right|, \quad z \in G, \xi \in C^{n} .
$$

The Kobayashi metric on $G$ (infinitesimal form) is defined by [8] $F_{K}(z, \xi)=\inf \left\{\alpha \mid \exists f \in G(\Delta)\right.$ with $\left.f(0)=z, f^{\prime}(0)=\xi / \alpha, \alpha>0\right\}$. For the definition of the Bergman metric see [1] or [4]. We take

$$
F_{B}(z, \xi)=\left(d s^{2}(z, \xi)\right)^{\frac{1}{2}}
$$

in the notation of [4].

We consider the boundary behavior of these metrics for fixed $\xi$. The notable features are (i) the different limiting behavior in tangential and normal directions (cf. Stein [9]), and (ii) the appearance of the Levi form as the limiting value of a quantity defined inside the domain.

THEOREM. Let $G$ be a (bounded) strongly pseudoconvex domain in $\boldsymbol{C}^{n}$ with $C^{2}$ boundary. Let $z_{0} \in \partial G$. Let $\varphi$ be a $C^{2}$ defining function for $\partial G$ such that $\left|\nabla_{z} \varphi\left(z_{0}\right)\right|=1$. Let $F(z, \xi)$ be either the Carathéodory or the Kobayashi metric on $G$. Then

$$
\lim _{z \rightarrow z_{0}} F(z, \xi) d(z, \partial G)=\frac{1}{2}\left|\nabla_{z} \varphi\left(z_{0}\right) \cdot \xi\right|=\frac{1}{2}\left|\xi_{N}\left(z_{0}\right)\right| .
$$

If $\nabla_{z} \varphi\left(z_{0}\right) \cdot \xi=0$, i.e. $\xi$ is a tangent vector to $\partial G$ at $z_{0}$, then

$$
\lim _{z \rightarrow z_{0} ; z \in \Lambda}(F(z, \xi))^{2} d(z, \partial G)=\frac{1}{2} \mathscr{L}_{\varphi, z_{0}}(\xi)=\frac{1}{2} \sum_{i, j=1}^{n} \frac{\partial^{2} \varphi}{\partial z_{i} \partial \bar{z}_{j}}\left(z_{0}\right) \xi_{i} \bar{\xi}_{j} .
$$

$d(z, \partial G)$ is the Euclidean distance to the boundary. $\nabla_{z} \varphi$ is the vector $\left(\partial \varphi / \partial z_{1}, \ldots, \partial \varphi / \partial z_{n}\right)$, and $\nabla_{z} \varphi\left(z_{0}\right) \cdot \xi=\sum_{i=1}^{n}\left(\partial \varphi / \partial z_{i}\right)\left(z_{0}\right) \xi_{i}=\xi_{N}\left(z_{0}\right)$ is the (complex) normal component of $\xi$ at $z_{0} . \Lambda$ in the second limit denotes a cone of arbitrary aperture with vertex at $z_{0}$ and axis the interior normal to $\partial G$.

AMS (MOS) subject classifications (1970). Primary 32D15, 32H15; Secondary 32E30, $32 \mathrm{H} 10$ 
For the Bergman metric a factor of $(n+1)^{1 / 2}$ appears in the first limit, and $(n+1)$ in the second. The first result was obtained by Diederich in [4] with the restriction $z \in \Lambda$. His methods easily give the second.

Full proofs will appear in the author's thesis and will be published elsewhere. E. Stein has informed me that some related results for the Carathéodory metric have been obtained by G. Henkin $[\mathbf{1 0}]$.

SKeTCH OF ProOF. As in [1], [4], and [5], we introduce suitable domains of comparison which pass through $z_{0}$ and in a sufficiently small neighborhood of $z_{0}$ lie inside or outside $G$. Explicit calculations are made for these domains. The reduction to local questions is made possible by (i) the monotonicity property of $F(z, \xi)$

$$
G \subset G^{\prime} \Rightarrow F_{G}(z, \xi) \geqq F_{G^{\prime}}(z, \xi), \quad z \in G ;
$$

(ii) (a) for the Carathéodory metric, an approximation theorem for bounded holomorphic functions due essentially to Diederich (Theorem 1 in [4]). Together with the introduction of a peak function at $z_{0}$, this gives

Proposition. Let $\varphi$, the function defining $\partial G$, be strictly pluri-subharmonic in a neighborhood $V$ of $z_{0} \in \partial G$. Let $\varepsilon>0$, and let $G_{1}=\{z \in V \mid \varphi(z)$ $\left.-\varepsilon\left\|z-z_{0}\right\|^{2}<0\right\}$. Then

$$
\varlimsup_{z \rightarrow z_{0}} \frac{F_{G_{1}}(z, \xi)}{F_{G}(z, \xi)} \leqq 1 .
$$

(b) For the Kobayashi metric, an estimate of Royden (Lemma 2 in [8]) which easily gives

Proposition. Let $G$ be a strongly pseudoconvex domain in $C^{n}$. Let $V$ be a neighborhood of $z_{0} \in \partial G$. Then

$$
\lim _{z \rightarrow z_{0}} \frac{F_{G \cap V}(z, \xi)}{F_{G}(z, \xi)}=1 .
$$

\section{BIBLIOGRAPHY}

1. S. Bergman, Uber die Kernfunktion und ihr Verhalten am Rande. I, II, J. Reine Angew. Math. 169 (1933), 1-42; 172 (1934), 89-128.

2. C. Carathéodory, Uber das Schwarze Lemma bei analytischen Funktionen von zwei komplexen Veränderlichen, Math. Ann. 97 (1926), 76-98.

3. - UUber die Geometrie der analytischen Abbildungen, die durch analytische Funktionen von zwei Veränderlichen vermittelt werden, Abh. Math. Sem. Univ. Hamburg. 6 (1928), 97-145.

4. K. Diederich, Das Randverhalten der Bergmanschen Kernfunktion und Metrik in streng pseudo-konvexen Gebieten, Math. Ann. 187 (1970), 9-36.

5. L. Hörmander, $L^{2}$ estimates and existence theorems for the o operator, Acta. Math. 113 (1965), 89-152. MR 31 \#3691.

6. S. Kobayshi, Hyperbolic manifolds and holomorphic mappings, Pure and Appl. Math., vol. 2, Marcel Dekker, 1970. MR 43 \# 3503. 
7. H.-J. Reiffen, Die differentialgeometrischen Eigenschaften der invarianten Distanzfunktion von Carathéodory, Sch. Math. Inst. Univ. Münster, Heft 26, 1963. MR 28 \# 1320.

8. H. L. Royden, Remarks on the Kobayashi metric. Several complex variables. II, Maryland 1970, 125-137; Springer-Verlag 1971.

9. E. M. Stein, Boundary behaviour of holomorphic functions of several complex variables, Princeton, Univ. Press, Princeton, N.J., 1972.

10. G. Henkin, Analytic polyhedra are not analytically equivalent to strictly pseudoconvex domains, Dokl. Akad. Nauk. SSSR (to appear).

Department of Mathematics, Princeton University, Princeton, New Jersey 08540 Current address: Department of Mathematics, Massachusetts Institute of Technology, Cambridge, Massachusetts 02139 